\title{
THE PROJECTIVE GEOMETRY ARISING FROM A HOLLOW MODULE
}

\author{
JEREMY E. DAWSON
}

(Received 5 May 1983)

Communicated by R. Lidl

\begin{abstract}
We discuss the projective geometry defined in terms of the hollow factor modules of a given module. In particular, we derive an explicit expression for the division ring obtained in coordinatizing such a projective geometry.
\end{abstract}

1980 Mathematics subject classification (Amer. Math. Soc.): 16 A 53, 05 B 35.

In [2] an independence structure was defined on the set of uniform submodules of a module, and was shown to be modular. Thus, if it is connected and of rank at least 3 , it corresponds naturally to a projective geometry, which is Desarguesian. The division rings obtainable by coordinatizing such projective geometries were discussed there in detail. Dually, in [3], an independence space, also modular, was defined on the set of hollow factor modules of a module. In this paper we discuss the division rings obtained by coordinatizing the associated projective geometries.

An independence structure $\mathscr{E}$ on a set $E$ is a collection of subsets (the independent sets), satisfying certain axioms, not unlike the properties of linear independence when $E$ is a subset of a vector space (see [7] for full details). The rank of $A \subseteq E$ is the cardinality of any maximal independent subset of $A$, and for $r$ finite, an $r$-flat is a maximal set of $\operatorname{rank} r$. If $\operatorname{rk}(A)=r$, then $[A]$ denotes the unique $r$-flat containing $A$, and we may write $[a, b]$ for $[\{a, b\}]$, for example. The 1-flats partition $E$; by collapsing each to a single element we get the simple independence space naturally associated with $\mathscr{E}$. A pair of elements $e, f \in E$ is connected if they are both contained in some circuit (minimal dependent set); connectedness is an

This paper was written while the author was at the National University of Singapore.

(1) 1984 Australian Mathematical Society 0263-6115/84 \$A2.00+0.00 
equivalence relation, the classes being called connected components. An independence structure is modular if $\operatorname{rk}(A)+\operatorname{rk}(B)=\operatorname{rk}(A \cup B)+\operatorname{rk}(A \cap B)$ for any flats $A, B \subseteq E$; some equivalent definitions are quoted in [2]. Further details are in [2], [7] etc.

Let $R$ be a ring with 1 ; all modules will be unitary left $R$-modules. A submodule $K$ of a module $M$ is small $\left(K \leqslant_{s} M\right)$ if $K+L=M \Rightarrow L=M$. A hollow module is not the sum of two proper submodules; let $H f(M)=\{N \leqslant M$; $M / N$ is hollow $\}$. We define $\mathscr{G} d(M) \subseteq \mathscr{P}(H f(M)$ ) (the set of subsets of $H f(M)$ ) by

(a) For $\left\{K_{1}, \ldots, K_{r}\right\} \subseteq H f(M),\left\{K_{1}, \ldots, K_{r}\right\} \in \mathscr{G} d(M)$ if, for each $l=1, \ldots, r$, $K_{l}+\bigcap_{j \neq l} K_{j}=M$. (In this case, for $\phi \subset J \subset I=\{1, \ldots, r\}, \bigcap_{i \in I \backslash J} K_{i}+\bigcap_{j \in J} K_{j}$ $=M$.)

(b) For $\left\{K_{i}: i \in I\right\} \subseteq H f(M),\left\{K_{i}: i \in I\right\}$ is in $\mathscr{G} d(M)$ if every finite subset of it is, according to (a).

The next theorem outlines the background to the present work.

THEOREM 1. (i) $\mathscr{G} d(M)$ is a modular independence structure on $H f(M)$.

(ii) If a connected component has rank at least 3 then its 1-flats and 2-flats form the points and lines of a projective geometry, which, if Desarguesian, is coordinatizable over a unique division ring $D$.

Proof. (i) is [3], Theorems 2.3 and 2.6. For (ii) combine standard results, as is done in [2], Theorem 9.

In examining when $\mathscr{G} d(M)$ is connected, we obtain the following result; its dual follows easily from [2], Lemma 10.

LEMMA 2. Let $N_{1}, N_{2} \in H f(M)$. Then $N_{1}$ and $N_{2}$ are connected if and only if $M / N_{1}$ and $M / N_{2}$ have isomorphic non-trivial factor modules.

Proof. Suppose $N_{1}$ and $N_{2}$ are connected. If $\left\{N_{1}, N_{2}\right\} \notin \mathscr{G} d(M)$, then $M /\left(N_{1}+N_{2}\right)$ is a common non-trivial factor module. Otherwise, since $\mathscr{G} d(M)$ is modular, there is a circuit $\left\{N_{1}, N_{2}, N^{\prime}\right\}$ for some $N^{\prime} \in H f(M)$. If $N=N^{\prime}+$ $\left(N_{1} \cap N_{2}\right)$, then by [3], Lemma $2.2, N<M$. Also, $N+N_{1}=N+N_{2}=N_{1}+N_{2}$ $=M$. We define a map $\theta: M / N_{1} \rightarrow M / N$ by $\left(m+N_{1}\right) \theta=n_{2}+N$, where $m=n_{1}+n_{2}, n_{i} \in N_{i}$. To show $\theta$ is well-defined, let $m \in N_{1}, m=n_{1}+n_{2}$, $n_{i} \in N_{i}$. Then $n_{2} \in N_{1} \cap N_{2} \leqslant N$. As $\operatorname{im} \theta=\left(N_{2}+N\right) / N=M / N$, we have $\left(M / N_{1}\right) / \operatorname{ker} \theta \cong M / N$. Similarly $M / N_{2}$ has a factor module isomorphic to $M / N$.

Conversely, let $\phi_{i}:\left(M / N_{i}\right) \rightarrow L \neq 0(i=1,2)$ be surjections. Define a map $\theta$ : $M \rightarrow L$ by $m \theta=\left(m+N_{1}\right) \phi_{1}+\left(m+N_{2}\right) \phi_{2}$. As $N_{2} \theta=\left(\left(N_{2}+N_{1}\right) / N_{1}\right) \phi_{1}=L$, 
$\operatorname{im} \theta=L$. Let $N=\operatorname{ker} \theta$, so $M / N \cong L$ and $N \in H f(M)$. As $N_{1} \cap N_{2} \leqslant N$, $\left(N_{1} \cap N_{2}\right)+N<M$ and $\left\{N_{1}, N_{2}, N\right\} \notin \mathscr{G} d(M)$. If $\left\{N_{1}, N_{2}\right\} \notin \mathscr{G} d(M)$ then clearly $N_{1}$ and $N_{2}$ are connected; otherwise, $N_{1}+N_{2}=M$ and it remains to show that $N_{1}+N=N_{2}+N=M$, whence $\left\{N_{1}, N_{2}, N\right\}$ is a circuit. Let $n_{1} \in N_{1}$; as $M=N_{1}+N_{2}$ and $\phi_{2}$ is onto, we can choose $n_{2} \in N_{2}$ such that $\left(n_{2}+N_{1}\right) \phi_{1}=$ $\left(n_{1}+N_{2}\right) \phi_{2}$. Then $n_{1}=\left(n_{1}-n_{2}\right)+n_{2} \in \operatorname{ker} \theta+N_{2}$, that is, $N_{1} \leqslant N+N_{2}$. Thus $M=N+N_{2}$, similarly $M=N+N_{1}$, and the result is shown.

We may make assumptions about the structure of $M$ while leaving the projective geometry, or at least one of its planes, unchanged.

LemMA 3. (i) Let $K \leqslant_{s} M$. Then $\mathscr{G} d(M / K)$ and $\mathscr{G} d(M)$ have isomorphic associated simple independence spaces.

(ii) Let $\left\{N_{1}, N_{2}, N_{3}\right\} \in \mathscr{G} d(M)$, let $K=N_{1} \cap N_{2} \cap N_{3}$. Then the associated simple independence space of $\mathscr{G}(M / K)$ is isomorphic to that of the subspace $\left[N_{1}, N_{2}, N_{3}\right]$ of $\mathscr{G} d(M)$.

Proof. Define a map $\theta: H f(M) \rightarrow H f(M / K) \cup\{M / K\}$ by $\theta(N)=$ $(N+K) / K$. For (i), as $K \leqslant_{s} M, N+K<M$; for (ii), $N+K<M$ if and only if $N \in\left[N_{1}, N_{2}, N_{3}\right.$ ], as follows from [3], Lemma 2.2. In this case, $N+K \in H f(M)$ and, equivalently, $(N+K) / K \in H f(M / K)$; also $[N]=[N+K]$ in $\mathscr{G} d(M)$. Thus, if $\left\{L_{i}: i \in I\right\} \subseteq H f(M)$ in (i), or $\left\{L_{i}: i \in I\right\} \subseteq\left[N_{1}, N_{2}, N_{3}\right]$ in (ii), then

$$
\begin{aligned}
\left\{L_{i}: i \in I\right\} \in \mathscr{G d}(M) & \Leftrightarrow\left\{L_{i}+K: i \in I\right\} \in \mathscr{G} d(M) \\
& \Leftrightarrow\left\{\left(L_{i}+K\right) / K: i \in I\right\} \in \mathscr{G} d(M / K) .
\end{aligned}
$$

THEOREM 4. The projective planes in the projective geometries of Theorem 1(ii) are precisely those arising from $M=H^{3}, H$ a hollow module; they are Desarguesian.

PROOF. Let $\left\{N_{1}, N_{2}, N_{3}\right\}$ be independent, in a connected component of $\mathscr{G} d(M)$. By Lemma 2 , let $K_{i} \geqslant N_{i}$ such that $M / K_{1} \cong M / K_{2} \cong M / K_{3} \cong H$ say, $H \neq 0$. Let $K=K_{1} \cap K_{2} \cap K_{3}, M^{\prime}=M / K$ and $K_{i}^{\prime}=K_{i} / K(i=1,2,3)$. Then, by Lemma 3(ii), the projective plane determined by $\left[N_{1}, N_{2}, N_{3}\right]\left(=\left[K_{1}, K_{2}, K_{3}\right]\right)$ is that of $\mathscr{G} d\left(M^{\prime}\right)$. As $\left(K_{1}^{\prime} \cap K_{2}^{\prime}\right)+K_{3}^{\prime}=M^{\prime},\left(K_{1}^{\prime} \cap K_{2}^{\prime}\right)+\left(K_{1}^{\prime} \cap K_{3}^{\prime}\right)=K_{1}^{\prime}$; as $K_{1}^{\prime} \cap K_{2}^{\prime} \cap K_{3}^{\prime}=0$, this sum is direct, as is $K_{1}^{\prime}+\left(K_{2}^{\prime} \cap K_{3}^{\prime}\right)=M^{\prime}$. This last also implies $K_{2}^{\prime} \cap K_{3}^{\prime} \cong M^{\prime} / K_{1}^{\prime} \cong H$; similar results give $M^{\prime}=K_{1}^{\prime} \cap K_{2}^{\prime}+K_{1}^{\prime} \cap K_{3}^{\prime}$ $+K_{2}^{\prime} \cap K_{3}^{\prime} \cong H^{3}$. Now $\mathscr{G} d\left(H^{4}\right)$ gives a projective geometry of rank 4 (dimension 3), necessarily Desarguesian; therefore its planes, which are isomorphic to $\mathscr{G} d\left(H^{3}\right)$ by Lemma 3(ii), are Desarguesian. Conversely, for $H$ hollow, $\mathscr{G} d\left(H^{3}\right)$ 
(with basis $\{(H, H, 0),(H, 0, H),(0, H, H)\})$ is connected by Lemma 2 , and therefore gives a projective plane, which is again Desarguesian.

We now describe the results of coordinatizing $\mathscr{G} d\left(H^{3}\right)$. Let us define the natural projections $p: H^{2} \rightarrow(H, 0)(=H \oplus 0)$, and $q: H^{2} \rightarrow(0, H)$.

THEOREM 5. The division ring which coordinatizes $\mathscr{G} d\left(H^{3}\right)$ is anti-isomorphic to the following.

$$
\begin{aligned}
& D=\left\{[M]: M \leqslant H^{2}, p(M)=H,(0, H) \nless M\right\} \text {, where } \\
& {[M]=[N] \Leftrightarrow M+N<H^{2} \Leftrightarrow(0, H) \nless M+N .} \\
& \quad 0_{D}=[(H, 0)], 0_{D}=[M] \Leftrightarrow q(M)<H, \text { and } 1_{D}=[\{(h, h): h \in H\}] . \\
& {[M]+[N]=[(M, N,+)] \text { and }[M] \times[N]=[(M, N, \times)], \text { where }} \\
& (M, N,+)=\left\{\left(m_{1}, m_{2}+n_{2}\right):\left(m_{1}, m_{2}\right) \in M,\left(n_{1}, n_{2}\right) \in N, m_{1}=n_{1}\right\} \text { and } \\
& (M, N, \times)=\left\{\left(m_{1}, n_{2}\right):\left(m_{1}, m_{2}\right) \in M,\left(n_{1}, n_{2}\right) \in N, m_{2}=n_{1}\right\} . \\
& \text { Also, }-[M]=\left[\left\{\left(m_{1},-m_{2}\right):\left(m_{1}, m_{2}\right) \in M\right\}\right] \text { and, for }[M] \neq 0_{D},[M]^{-1}= \\
& {\left[\left\{m_{2}, m_{1}\right\}:\left(m_{1}, m_{2}\right) \in M\right] \text {. }}
\end{aligned}
$$

Proof. We follow the coordinatization rule of [5], p. 209. Let the coordinate line $D \cup\left\{\infty_{D}\right\}$ be $[(H, 0, H),(0, H, H)]$. If $N \in[(H, 0, H),(0, H, H)]$ then $N+$ $(0,0, H)<H^{3}$ and so $[N]=[N+(0,0, H)]$. We will therefore consider $D \cup$ $\left\{\infty_{D}\right\}$ as the set of 1-flats of $\mathscr{G} d\left(H^{2}\right)$, under the well-defined 1-1 correspondence $[N] \leftrightarrow[N \oplus H]\left(N \in h f\left(H^{2}\right), N \oplus H \in H f\left(H^{3}\right)\right)$. Choose $0_{D}$ and $1_{D}$ as stated, and $\infty_{D}=[(0, H)] /$ Since, for $M, N \in H f\left(H^{2}\right),[M]=[N]$ when $M+N<H^{2}$, we have $[M]=0_{D}$ when $q(M)<H$ and $[M]=\infty_{D}$ when $p(M)<H$. Let $M \leqslant H^{2}$ such that $p(M)=H$. Then $M<H^{2} \Leftrightarrow(0, H) \nless M$, and in this case $M \in$ $H f\left(H^{2}\right)$, by [3], Lemma 3.5(i), since $M+(0, H)=H^{2}$. Likewise $M+N<H^{2}$ $\Leftrightarrow(0, H) \nless M+N$. Thus $D$ is as stated. The coordinatization procedure then gives the operations. We omit the details, but the following Lemma is used in the construction.

Lemma 6. Let $[A, B]$ and $[C, D]$ be two distinct lines (with $A, B, C, D \in$ $\left.H f\left(H^{3}\right)\right)$. Then $[A, B] \cap[C, D]=[N]$, where $N=A \cap B+C \cap D$.

Proof. As $\operatorname{rk}\left(\mathscr{G} d\left(H^{3}\right)\right)=3,\{A, B, C, D\}$ contains a circuit, which is not contained in either $\{A, B\}$ or $\{C, D\}$. Therefore, by [3], Lemma $2.2, N<H^{3}$. We show $H^{3} / N$ is hollow. Suppose $N \leqslant K^{\prime}, L^{\prime}<H^{3}$. Let $K \geqslant K^{\prime}, L \geqslant L^{\prime}$ such that $K, L \in H f\left(H^{3}\right)$, by [3], Theorem 2.5. As $K \geqslant N \geqslant A \cap B$, and similarly, we 
have $\{K, L\} \subseteq[A, B] \cap[C, D]$; as

$$
\begin{aligned}
\operatorname{rk}([A, B] \cap[C, D]) & =\operatorname{rk}([A, B])+\operatorname{rk}([C, D])-\operatorname{rk}([A, B, C, D]) \\
& =2+2-3=1,
\end{aligned}
$$

$[K]=[L] ;$ that is, $K+L<H^{3}$. Thus $N \in H f\left(H^{3}\right)$, and clearly $N \in[A, B] \cap$ $[C, D]$.

Naturally, it can be verified directly that $D$ is a division ring. Clearly, $(M, N,+)$ and $(M, N, \times)$ are submodules of $H^{2}$ which project onto $(H, 0)$. As $(0, H)$ is hollow, $(M, N,+) \cap(0, H)=M \cap(0, H)+N \cap(0, H)<(0, H)$, so $(M, N,+)<H^{2}$. To check that $(M, N, \times)<H^{2}$ requires the following interesting lemma.

Lemma 7. Let $N^{\prime} \leqslant N<H^{2}$, such that $p(N)=q(N)=H$. Then $p\left(N^{\prime}\right)=H \Leftrightarrow$ $q\left(N^{\prime}\right)=H$, and in this case $\left[N^{\prime}\right]=[N]$.

Proof. Suppose $p\left(N^{\prime}\right)=H$. Then $N^{\prime} \in H f\left(H^{2}\right)$, and since $N^{\prime}+N=N<H$, $\left[N^{\prime}\right]=[N]$. As $q(N)=H,\left[N^{\prime}\right]=[N] \neq 0_{D}$, so $q\left(N^{\prime}\right)=H$. The converse is by symmetry.

Consider $(M, N, \times)$ where $[M],[N] \in D,[N] \neq 0_{D}$. Let $N^{\prime}=\left\{\left(n_{1}, n_{2}\right) \in N\right.$ : $\left.\left(0, n_{1}\right) \in M\right\}$. By the lemma, we get

$$
(0, H) \leqslant(M, N, \times) \Rightarrow q\left(N^{\prime}\right)=H \Rightarrow p\left(N^{\prime}\right)=H \Rightarrow(0, H) \leqslant M,
$$

which is not so. Thus $(M, N, \times)<H^{2}$. It is easy to show that calculating $[M]-[N]$ gives $0_{D}$ if and only if $[M]=[N]$, and this leads to a proof that the operations are well-defined. The remaining details are easy to verify (noting that to show, say, $[A]=[B]$, it is enough to show that, for example, $A \geqslant B$ ).

We turn now to some special cases. Since a hollow module is either cyclic or not finitely generated, we consider $H$ cyclic, $H=R h$. Let $H \cong R / I, I$ a left ideal of $R$. For $[M] \in D, p(M)=H$ and so we may choose $(h, m) \in M$. Then by Lemma $7,[R(h, m)]=[M]$, and it also follows that $R m=H$ if and only if $q(M)=H$. If we denote $[R(h, m)]$ by $\langle m\rangle$, we get

$$
\begin{aligned}
D & =\left\{\langle m\rangle: m \in H, R(h, m)<H^{2}\right\}, \text { where } \\
\langle m\rangle & =\langle n\rangle \Leftrightarrow R(h, m)+R(h, n)<H^{2}, \\
0_{D} & =\langle 0\rangle ; 0_{D}=\langle n\rangle \Leftrightarrow R n<H ; 1_{D}=\langle h\rangle, \\
\langle m\rangle \pm\langle n\rangle & =\langle m \pm n\rangle, \\
\langle m\rangle \times\langle n\rangle & =\langle m\rangle \text { and }\langle n\rangle^{-1}=\langle s h\rangle, \quad \text { where } m=r h \text { and } h=s n .
\end{aligned}
$$


Note that, if $I=\operatorname{Ann}(h)$, then $R(h, m)<H^{2} \Leftrightarrow I m<H$.

However, the case where $H$ is cyclic is always covered by the following Theorem (see [4], Corollary 2.2). Let the division ring $D$ described in Theorem 5 be called $D d\left(H^{3}\right)$.

THEOREM 8. If $H$ is hollow and $K<H$, then $D d\left(H^{3}\right) \cong D d\left((H / K)^{3}\right)$. If $H$ also has a maximal submodule (that is, $J(H)<H)$, then $D d\left(H^{3}\right) \cong \operatorname{En}(H / J(H))$.

Proof. As $H$ is hollow, $K \leqslant_{s} H, K^{3} \leqslant_{s} H^{3}$ (by [1], 5.20(1)), and, from Lemma 3(i), $D d\left(H^{3}\right) \cong D d\left(H^{3} / K^{3}\right) \cong D d\left((H / K)^{3}\right)$. If $H$ has a maximal submodule, then it is unique, since $H$ is hollow, and so $J(H)$ is maximal. Let $N=H / J(H)$ and, as $N$ is simple, let $N=R h \cong R / I$.

Define $f: \operatorname{En}(N) \rightarrow D d\left(N^{3}\right)$ by $f(\psi)=\langle h \psi\rangle$. Now $\psi=0 \Leftrightarrow h \psi=0 \Leftrightarrow\langle h \psi\rangle$ $=0_{D}$ as $N$ is simple. To show $f$ is onto, let $\langle m\rangle \in D d\left(N^{3}\right)$. Thus $\operatorname{Im}<N$, so $\operatorname{Im}=0$, and we may define $\psi \in \operatorname{En}(N)$ by $(r h) \psi=r m$. Also, for $\psi \in \operatorname{En}(N)$, $I(h \psi)=(I h) \psi=0$. Clearly $f$ preserves the operations, and so is an isomorphism.

It can be verified that in the case where $H$ is cyclic, $H=R h$, then $\langle h \psi\rangle \leftrightarrow \psi$ is an isomorphism from $D d\left(H^{3}\right)$ to $\operatorname{En}(H / J(H))$. This last theorem is the dual of part of [2], Theorem 15. The proof is not similar because projective covers need not exist.

Suppose that in fact $H$ does have a projective cover $P$, that is, $H \cong P / K$, $K \leqslant_{s} P$. Then $P$ is also hollow. By [1], 17.14, $P$ has a maximal submodule $M$; as $K \leqslant_{s} P, K \leqslant M$, and so $M / K$ is maximal in $H$. Thus Theorem 8 applies. Also, $M / K$ and hence $M$ are unique maximal submodules, of $H$ and $P$ respectively, so $P / J(P) \cong H / J(H)$. From [1], 17.12 and 17.10 we have $\operatorname{En}(P / J(P)) \cong$ $\operatorname{En}(P) / J(\operatorname{En}(P))$. Thus $D d\left(H^{3}\right) \cong \operatorname{En}(P) / J(\operatorname{En}(P))$, corresponding to [2], Theorem 14. It follows from this that $\operatorname{En}(P)$ is (quasi-)local, for $P$ hollow projective; this is also shown in [6], Proposition 4.1 and Theorem 4.2, which characterize hollow projective modules (see also [1], 17.19).

There remain the hollow modules with no maximal submodule (and therefore no projective cover). We look at the example $H=\mathbf{Z}_{p^{\infty}}$ (a $\mathbf{Z}$-module). This is hollow, and has no maximal submodule, since all proper submodules are finitely generated (indeed finite and cyclic), see [4], Section 5.

Let $N \in H f\left(H^{2}\right)$, with $p(N)=H$. As $(0, H) \nless N, N \cap(0, H)=\mathbf{Z}\left(0,1 / p^{e}\right)$ for some $e \geqslant 0$. Thus, if $\left(1 / p^{i}, m\right)$ and $\left(1 / p^{j}, n\right)(i>j>0)$ are in $N$, then $p^{i-j}\left(1 / p^{i}, m\right)-\left(1 / p^{j}, n\right) \in \mathbf{Z}\left(0,1 / p^{3}\right)$. So, if

$$
m \in \frac{a_{k}}{p^{i-k}}+\frac{a_{k+1}}{p^{i-k-1}}+\cdots+\frac{a_{i-e-1}}{p^{e+1}}+\mathbf{Z}\left(\frac{1}{p^{e}}\right)
$$


then

$$
n \in \frac{a_{k}}{p^{j-k}}+\frac{a_{k+1}}{p^{j-k-1}}+\cdots+\frac{a_{j-e-1}}{p^{e+1}}+\mathbf{Z}\left(\frac{1}{p^{e}}\right) .
$$

Let us therefore describe $N$ by the power series expression $a_{k} p^{k}+a_{k+1} p^{k+1}+$ $\cdots\left(0 \leqslant a_{i}<p, a_{k} \neq 0\right)$. Any coefficient $a_{l}$ is determined by choosing $j>l+e$ and $\left(1 / p^{j}, n\right) \in N$; then $a_{l}$ appears in the expression for $n . D d\left(H^{3}\right)$ is the set of such power series expressions, addition and multiplication being natural; it is the $p$-adic completion of the rationals.

Another example would be $H=\mathbf{Z}[1 / p]$. However, since $\mathbf{Z}_{p^{\infty}}=\mathbf{Z}[1 / p] / \mathbf{Z}$, the same division ring arises, by Theorem 8 .

I wish to thank Dr. M. Keating for some helpful discussions.

\section{References}

[1] F. W. Anderson \& K. R. Fuller, Rings and categories of modules (Springer-Verlag, New York, 1973).

[2] J. E. Dawson, 'Independence spaces and uniform modules', European J. Combinatorics, to appear.

[3] J. E. Dawson, 'Independence spaces on the submodules of a module', European J. Combinatorics, to appear.

[4] P. Fleury, 'Hollow modules and local endomorphism rings', Pacific J. Math. 53 (1974), 379-385.

[5] G. Grătzer, General lattice theory (Birkhaüser, Basel, 1978).

[6] R. Ware, 'Endomorphism rings of projective modules', Trans. Amer. Math. Soc. 155 (1971), 233-256.

[7] D. J. A. Welsh, Matroid theory (Academic Press, London, 1976).

CSIRO Division of Mathematics and Statistics

P. O. Box 218

Lindfield, NSW 2070

Australia 